\title{
The evacuation plan design under uncertain times of transportation
}

\author{
J. Janáček \\ Faculty of Management Science and Informatics, University of Žilina, \\ Slovak Republic
}

\begin{abstract}
When the population of a given set of municipalities are endangered by some threat, casualties can be avoided by evacuation of the endangered population to safer refuges. When an evacuation plan is designed, vehicles are assigned to the evacuated municipalities so that the entire population is evacuated in a minimal time. Nevertheless, the transportation times in the associated road network depend on many unpredictable circumstances and this makes them uncertain. This paper deals with an approach, which processes the uncertainty making use of the theory of fuzzy sets. Furthermore, the presented approach uses a special feature of professional IP-solver to find the optimal solution of the evacuation plan design problem in acceptable computation time.
\end{abstract}

Keywords: evacuation plan, vehicle assignment, min-max problem, uncertain transportation time, fuzzy technique.

\section{Introduction}

The evacuation problem formulation and attempt for solving it are motivated by a situation in which some populated area is endangered by an incoming disaster. The endangered population is concentrated at several dwelling places of the area and there are some refuges located outside the area to where the population should be evacuated. It can be assumed that a preceding consideration has been done and that the dwelling places are assigned to the individual refuges so that the whole population of one dwelling place should be evacuated to exactly one of the refuges.

For the evacuation, there are at its disposal some homogeneous fleets of vehicles. Each fleet is characterized by its location, number of vehicles and 
vehicle capacity, where the vehicle capacity is measured by the number of persons which can be transported by one tour of the vehicle.

The evacuation problem consists in vehicle route determination so that the population is evacuated from the dwelling places to the predetermined refuges in the minimal time. The time of evacuation is considered as a period between the start of the evacuation and the time when the last person reaches its predestined refuge (Teichmann [1]).

As shown in Janáček and Sibila [2], the problem represents hard combinatorial problem, the model of which is non-linear even if a complexity of vehicle route is limited. There was considered that a vehicle route can take only the following form. The route starts at a fleet location, continues to a given dwelling place, picks up a portion of the evacuated population and takes them to the predetermined refuge. Being at the refuge, the route can be enlarged only by return to the given dwelling place and taking another portion of the population to the same refuge. This cycle can be repeated several times within the route. This problem was solved in Janáček and Sibila [2] for known transportation times by model linearization and successive use of a professional IP-solver. In Janáček [3], an iterative approach to this problem solving was presented. This approach proved to be much more effective in connection with the IP-solver application. In this paper, we deal with a more real situation which can arise during the period of evacuation. The previous approaches assumed that the times for traversing from a vehicle depot to an evacuated place and from an evacuated place to its predetermined refuge are given by known real values. On the contrary to this preposition, we consider that these values are uncertain and triangle fuzzy numbers describe them. In this contribution, we suggested two approaches to the evacuation problem with fuzzy times, we verify them and report results of numerical experiments to show usability of the approaches.

\section{Modelling and solving of the crisp evacuation problem}

In accordance with Janáček [3], we use the notation, where $I$ denotes a set of considered fleets of vehicles. The symbol $N_{i}$ denotes the number of vehicles belonging to the fleet $i$ and $K_{i}$ let be the capacity of one vehicle of this fleet. The evacuated dwelling places form a set $J$, where $b_{j}$ for $j \in J$ denotes the number of persons which must be evacuated from the dwelling place $j$ to the predetermined refuge. Furthermore, let $t_{i j}$ denote the time, which is necessary for a vehicle to traverse the distance between the locations of the fleet $i$ and the dwelling place $j$. In addition, let us denote by $s_{j}$ the time necessary for traversing the distance between the dwelling place $j$ and the predetermined refuge.

The objective is to determine a route of each vehicle so that all the population is evacuated and the longest route is minimal Janáček and Sibila [2]. Coming out of the assumption on the possible form of a vehicle, we introduce the variable $z_{i j} \in\{0,1\}$ defined for each pair of fleet $i$ and dwelling place $j$. This variable takes the value of 1 if and only if the positive number of vehicles of fleet $i$ is assigned to the municipality $j$. 
Taking into account the special form of vehicle route, it is sufficient to introduce only three series of decision variables to model all the decisions, which determine a system of vehicle routes.

The variable $q_{i j} \in Z^{+}$models the decision on number of vehicles from the fleet $i$ assigned to the dwelling place $j$.

The variable $x_{i j} \in Z^{+}$models the decision on the maximal number of visits of each vehicle from the fleet $i$ assigned to the place $j$ at this place. The multiple of $K_{i}$ and $x_{i j}$ gives the number of persons, which can be evacuated from the dwelling place $j$ by one vehicle from the fleet $i$.

The variable $z_{i j} \in\{0,1\}$ is an auxiliary variable, which takes the value of one, if any vehicle from the fleet $\mathrm{i}$ visits the dwelling place $j$. The last introduced variable is $T \geq 0$. This variable represents an upper limit on time of vehicle routes. After these preliminaries a model of the evacuation problem can be written as follows:

Minimize $T$

$$
\begin{array}{cc}
\text { Subject to } & \left(t_{i j}-s_{j}\right) z_{i j}+2 s_{j} x_{i j} \leq T \quad \text { for } i \in I, j \in J \\
& x_{i j} \leq P z_{i j} \quad \text { for } i \in I, j \in J \\
& \sum_{j \in J} q_{i j} \leq N_{i} \quad \text { for } i \in I \\
& \sum_{i \in I} K_{i} q_{i j} x_{i j} \geq b_{j} \quad \text { for } j \in J \\
& z_{i j} \in\{0,1\} \quad \text { for } i \in I, j \in J \\
& x_{i j} \in Z^{+} \quad \text { for } i \in I, j \in J \\
& T \geq 0 \\
& q_{i j} \in Z^{+} \quad \text { for } i \in I, j \in J
\end{array}
$$

The constraints (2) assure that the travelling time of a vehicle from the fleet assigned to the dwelling place $j$ is less or equal to the upper bound $T$ as the lefthand-side of (2) represents the maximal time of vehicle from the fleet $i$ assigned to the dwelling place $j$. The constraints (3) are link-up constraints, which cause that the variable $z_{i j}$ is equal to one whenever the variables $x_{i j}$ is greater or equal to one. The constraints (4) assure that the total number of designated vehicles of the fleet $i$ does not exceed the number $N_{i}$.

The constraints (5) ensure that each dwelling place $j$ is provided with a sufficient capacity, which enables to evacuate all population $b_{j}$ of the dwelling place $j$. These constraints are non-linear with respect of variables $x_{i j}$ and $q_{i j}$, what prevents from making use of commercial IP-solvers, which can deal with linear constraints only. This obstacle motivated the following approach (Janáček [3]) to the evacuation problem.

The suggested iterative method resembles the Tanaka-Asai's method used in the fuzzy optimization to master non-linearity connected with using level of satisfaction as a variable (Teodorovič and Vukadinovič [4]). The method is based on fixing the variable $T$ of the model (1)-(9) at an integer value $T^{f i x}$ and on searching for a feasible solution of the resulting model, which becomes linear. If 
the upper bound on route time is fixed at $T^{f i x}$, then the maximal value of the variable $x_{i j}$ can be determined from the constraint (2), which represents the only upper bound on this variable. The maximal value $P_{i j}\left(T^{f i x}\right)$ can be obtained from (10).

$$
P_{i j}\left(T^{f i x}\right)=\left\lfloor\frac{T^{f i x}-t_{i j}+s_{j}}{2 s_{j}}\right\rfloor \text { if } t_{i j}+s_{j} \leq T^{f i x} \text { and } 0 \text { otherwise }
$$

The variable $z_{i j}$ can be set at the value of 1 , if $P_{i j}\left(T^{f i x}\right) \geq 1$ and at the value of 0 otherwise without loss of generality. Then searching for feasible solution of the problem (1)-(9) is reduced to search for a feasible solution of the problem (11)(13).

$$
\begin{gathered}
\text { Subject to } \sum_{j \in J} q_{i j} \leq N_{i} \quad \text { for } i \in I \\
\sum_{i \in I} K_{i} P_{i j}\left(T^{f i x}\right) q_{i j} \geq b_{j} \quad \text { for } j \in J \\
q_{i j} \in Z^{+} \text {for } i \in I, j \in J
\end{gathered}
$$

To find a feasible solution of the linear problem described by the constraints (11)-(13), a commercial IP-solver [5, 6] can be easily used. The solving procedure of the IP-solver uses branch-and-bound method with premature stopping rule for the case, when an integer solution is met or given computational time limit is exceeded. This procedure is also able to complete the whole searching process if no solution exists and to refer to this fact. As the used optimisation software environment XPRESS-IVE is equipped with the programming language Mosel, there can be easily implemented bisection method, which starts from two values $T^{\min }$ and $T^{\max }$. The value $T^{\text {min }}$ is a time, in which the endangered area cannot be evacuated and the value $T^{\text {max }}$ is a time for which a feasible solution of the problem (11)-(13) exists. This implementation of bisection method can make use the solving procedure of IP-solver and it is able to find the lowest value of $T^{f i x}$, for a feasible solution of (11)-(13) exists with a demanded precision. This resulting time $T^{\text {ppt }}$ is the optimal time of evacuation under pre-condition that the used solving procedure completes its searching process. If this process is prematurely stopped, the bisection method becomes a heuristic. This bisection method is referred as DejTopt in reminder of this paper.

\section{Fuzzy approach to the evacuation problem}

In this case, we assume that the travelling times $t_{i j}$ and $s_{j}$ are uncertain and that triangular fuzzy numbers describe these uncertain values. It means that an uncertain value, e.g. $\boldsymbol{t}_{i j}$, is described by a membership function $\mu_{t i j}$ defined on the universe $R$ of real numbers. This membership function gives to each $t \in R$ a weight $\mu_{t i j}(t)$ from the range $\langle 0,1\rangle$, to express the power with which the time $t$ belongs to the fuzzy number $\boldsymbol{t}_{i j}$. If $\boldsymbol{t}_{i j}$ is a triangular fuzzy number, the graph of 
associated membership function has special form of triangle. It means that the membership function is defined by three values $t_{i j}^{1}, t^{2}{ }_{i j}$ and $t_{i j}^{3}$, which are called left value, main value and right value respectively. The definition of the membership function follows.

$$
\begin{gathered}
\mu_{\mathrm{t}_{i j}}(t)=0 \quad \text { if } t \leq t_{i j}^{1} \text { or } t \geq t_{i j}^{3} \\
\mu_{\mathrm{t}_{i j}}(t)=\left(t-t_{i j}^{1}\right) /\left(t_{i j}^{2}-t_{i j}^{1}\right) \quad \text { if } t \in<t_{i j}^{1}, t_{i j}^{2}> \\
\mu_{\mathrm{t}_{i j}}(t)=\left(t_{i j}^{3}-t\right) /\left(t_{i j}^{3}-t_{i j}^{2}\right) \quad \text { if } t \in<t_{i j}^{2}, t_{i j}^{3}>
\end{gathered}
$$

The shape of the membership function $\mu_{t i j}$ is depicted in fig. 1 .

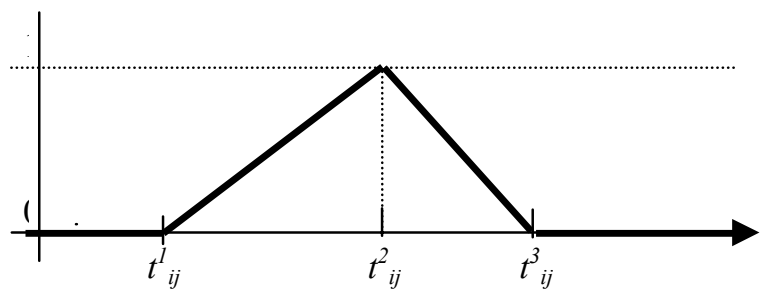

Figure 1: The membership function of a triangular fuzzy number $\boldsymbol{t}_{i j}$.

The same form of triangular fuzzy number is assumed for the time $s_{j}$.

The core of fuzzy approach to a general mathematical programming problem consists in determination of the highest level of satisfaction $h$, for which the associated constraints are satisfied and the objective function value belongs to a fuzzy set of satisfactorily small values of objective function. The fuzzy set of satisfactorily small values is usually constructed from two real values $F^{l}$ and $F^{2}$, where $F^{l}$ corresponds to the optimal objective function value for the most favourite case of the problem coefficients and $F^{2}$ corresponds to the optimal objective function value for the main values or for the least favourite case of the problem coefficients. The membership function $\mu_{F}(T)$ is shown in fig. 2.

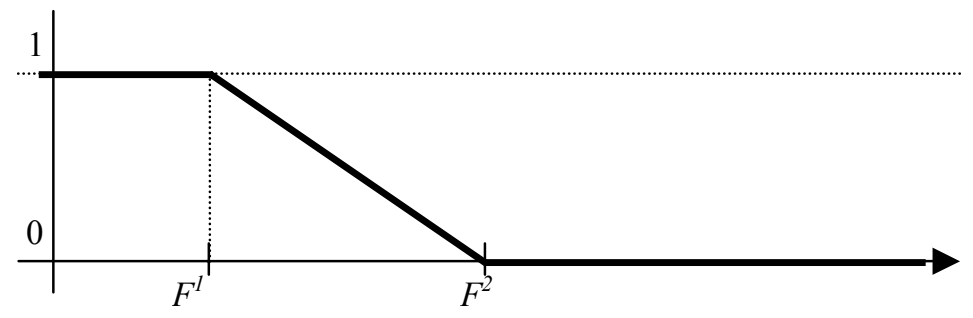

Figure 2: The membership function of a fuzzy set of sufficiently small values.

The constraint ensuring that the objective function value, say time $T$ of evacuation in our case, belongs to the sufficiently small values of evacuation times at a level of satisfaction $h$, follows. 


$$
T \leq h F^{1}+(1-h) F^{2}
$$

Now, let us focus on the way, how the time uncertainty influences the model (11)-(13). The only model coefficient influenced by uncertain times is $P_{i j}\left(T^{f i x}\right)$. This coefficient takes a form of fuzzy value, which is more complicated than a simple triangular fuzzy number. Even if the times $\boldsymbol{t}_{i j}$ and $\boldsymbol{s}_{j}$ are positive triangular fuzzy numbers given by the triples $<t_{i j}^{1}, t_{i j}^{2}, t_{i j}^{3}>$ and $\left\langle s_{j}^{1}, s_{j}^{2}, s_{j}^{3}>\right.$, the fuzzy value $\boldsymbol{P}_{i j}(T)$ is defined as

$$
\mathbf{P}_{i j}(T)=\left\lfloor\frac{T-\mathbf{t}_{i j}+\mathbf{s}_{j}}{2 \mathbf{s}_{j}}\right\rfloor=\left\lfloor\frac{\mathbf{n}_{i j}}{\mathbf{d}_{j}}\right\rfloor \text { if } \boldsymbol{t}_{i j}+\boldsymbol{s}_{j} \leq T \text { and } 0 \text { otherwise }
$$

It means that $\boldsymbol{P}_{i j}(T)$ is not only a general fuzzy number, but, in addition, it is conditioned by the fuzzy condition $\boldsymbol{t}_{i j}+\boldsymbol{s}_{j} \leq T$.

In accordance to max-min principle (Ramík and Vlach [7]), if a constraint "great or equal to" from the set of constraints (12) is evaluated at a level of satisfaction $h$, the most right value of $\boldsymbol{P}_{i j}(T)$ at the level $h$ is taken into account. Let us denote this most right value of $\boldsymbol{P}_{i j}(T)$, which belongs to this fuzzy value with membership function value at least $h$, as $P_{i j}^{R}(T, h)$. Then, we claim that constraints (12) with fuzzy value $\boldsymbol{P}_{i j}(T)$ on the place of $P_{i j}(T)$ holds at level $h$ if constraints (19) hold.

$$
\sum_{i \in I} K_{i} P_{i j}^{R}\left(T^{f i x}, h\right) q_{i j} \geq b_{j} \quad \text { for } j \in J
$$

Let us derive the value of $P_{i j}^{R}(T, h)$ from the triangular fuzzy times $\boldsymbol{t}_{i j}$ and $\boldsymbol{s}_{j}$. Let us denote by $\boldsymbol{e}_{i j}$ the triangular fuzzy number $\boldsymbol{t}_{i j}+\boldsymbol{s}_{j}$. If the inequality $\boldsymbol{e}_{i j} \leq T$ is said that it holds at level $h$, then it means that the most left value of $\boldsymbol{e}_{i j}$, which belongs to this fuzzy number with membership function value at least $h$, is less or equal to $T$. If we denote $e^{L}{ }_{i j}(h)$ this most left value, then we say that inequality $\boldsymbol{e}_{i j} \leq T$ holds at the level of satisfaction $h$ if $e^{L} i j(h) \leq T$ holds. In accordance to $\alpha$ cut approach, the function $e^{L}{ }_{i j}(h)$ is given by the expression (20).

$$
e_{i j}^{L}(h)=(1-h)\left(t_{i j}^{1}+s_{j}^{1}\right)+h\left(t_{i j}^{2}+s_{j}^{2}\right)
$$

Now, let us concentrate on the ratio $\boldsymbol{n}_{i j} / \boldsymbol{d}_{j}$ of the triangular fuzzy numbers $\boldsymbol{n}_{i j}=T-\boldsymbol{t}_{i j}+\boldsymbol{s}_{j}$ and $\boldsymbol{d}_{j}=2 \boldsymbol{s}_{j}$. Using the $\alpha$-cut approach and taking into account that $n^{R}{ }_{i j}(h)>0$ follows from $e^{L}{ }_{i j}(h) \leq T$, it can be inferred that the most right value belonging to $\boldsymbol{n}_{i j} / \boldsymbol{d}_{j}$ at the level of satisfaction $h$ is $n^{R}{ }_{i j}(h) / d^{L}{ }_{j}(h)$. Then, it follows that $P_{i j}^{R}(T, h)$ is given by the expression (21).

$$
P_{i j}^{R}(T, h)=\left\lfloor\frac{n_{i j}^{R}}{d_{j}^{L}}\right\rfloor=\left\lfloor\frac{(1-h)\left(T-t_{i j}^{1}+s_{j}^{3}\right)+h\left(T-t_{i j}^{2}+s_{j}^{2}\right)}{(1-h) 2 s_{j}^{1}+h 2 s_{j}^{2}}\right\rfloor \text { if } e_{i j}^{L}(h) \leq T
$$

and $P_{i j}^{R}(T, h)=0$ otherwise. 
Now, it is possible to answer the question whether there is any integer solution $\boldsymbol{q}$ with an evacuation time so that $T$ is small enough and constraints (11) and (12) are satisfied at level of satisfaction $h$. It is sufficient to solve the following linear program:

\section{Minimize T}

Subject to (11), (19), (13) and (17).

As we have designed the procedure DejTopt, which is able to solve the problem: Minimize $T$ subject to (11), (19), (13), we can implement the TanakaAsai's method in accordance to the following steps, where $\varepsilon$ is a demanded precision of the maximal level of satisfaction.

0 . Set $h_{\min }:=0, h_{\max }:=1$.

1. Repeat the steps 2,3 and 4 until $h_{\max }-h_{\min }<\varepsilon$ is met.

2. Set $h:=\left(h_{\max }+h_{\min }\right) / 2$.

3. Apply procedure DejTopt on the problem: (11), (16) and (13) for given $T^{\text {max }}$ and $T^{\text {min }}$.

4. If no solution $q$ exists or the associated $T$ does not satisfy the constraint (14),

set $h_{\max }:=h$, otherwise set $h_{\max }:=h$ and update the best found solution $\boldsymbol{q}^{\text {best }}$ and $T^{\text {best }}$.

This implementation will be referred as FuzzyTopt_1. To avoid the inside iterative process performed in the procedure DejTopt, we suggested and implemented another procedure. This procedure called FuzzyTopt 2 determines $T$ for a given level of satisfaction $h$ as the right-hand-side of inequality (17). Then $P^{R}{ }_{i j}(T, h)$ is computed in the step 3 of the above/mentioned algorithm and the branch-and-bound searching process of XPRESS-IVE is used to find a feasible solution of (11), (19) and (13). The both procedures were tested and the numerical results are reported in the next section.

\section{Numerical experiments}

To verify the both designed procedures FuzzyTopt_1 and FuzzyTopt_2, we used the general optimisation software environment XPRESS-IVE (see $[5,6])$. We made use of that system includes a branch-and-cut method equipped with premature stopping rules. In all our experiments, we set the stopping rule so that the branch-and-cut search terminates if the first integer solution is found or if the time of search exceeds ten seconds.

The pool of solved instances was overtaken from Janáček [3], where ten different instances of the evacuation problem were for disposal. One of the instances referred as "Hradza" comes out of a possible emergency situation, which could occur if the dam Liptovska Mara breaks. Then, under given conditions, 26 communities would have to be evacuated to 26 predestined refuges. 
Table 1: $\quad$ Parameters of solved instances.

\begin{tabular}{|c|c|c|c|c|c|}
\hline Instance: & Bratislava & $\begin{array}{c}\text { Dubnica nad } \\
\text { Váhom }\end{array}$ & Hrádza & Košice & $\begin{array}{c}\text { Liptovský } \\
\text { Mikuláš }\end{array}$ \\
\hline No. of F. & 14 & 10 & 16 & 13 & 9 \\
\hline No. of EP. & 25 & 25 & 26 & 25 & 25 \\
\hline$T^{\max }[\mathrm{min}]$ & 100 & 400 & 200 & 100 & 250 \\
\hline$F^{I}[\mathrm{~min}]$ & 54 & 242 & 54 & 62 & 159 \\
\hline$F^{2}[\mathrm{~min}]$ & 81 & 362 & 81 & 92 & 238 \\
\hline Instance: & Leopoldov & Michalovce & Nitra & $\begin{array}{c}\text { Nové } \\
\text { Zámky }\end{array}$ & Púchov \\
\hline No. of F. & 11 & 10 & 10 & 9 & 12 \\
\hline No. of EP. & 25 & 25 & 25 & 25 & 25 \\
\hline$T^{\max }[\mathrm{min}]$ & 100 & 500 & 200 & 630 & 150 \\
\hline$F^{I}[\mathrm{~min}]$ & 62 & 313 & 112 & 387 & 93 \\
\hline$F^{2}[\mathrm{~min}]$ & 93 & 469 & 168 & 570 & 139 \\
\hline
\end{tabular}

For this evacuation, 411 vehicles located at three locations are available. These vehicles were partitioned into 16 homogenous fleets, which differ either in location or in vehicle capacity. The other instances were formulated in nine areas of the Slovak Republic in the similar way. These instances are called by names of the biggest towns of those areas. The numbers and capacities of available vehicles were generated similarly to the instance "Hradza". All values of $T^{\text {max }}$ necessary for starting of DejTopt were also overtaken from Janáček [3] and are reported in table 1 . The time $T^{\text {min }}$ was set to the value of zero in all instances.

Table 2: The comprehensive results obtained by the procedure FuzzyTopt_1.

\begin{tabular}{|c|c|c|c|c|c|}
\hline Instance: & Bratislava & $\begin{array}{c}\text { Dubnica nad } \\
\text { Váhom }\end{array}$ & Hrádza & Košice & $\begin{array}{c}\text { Liptovský } \\
\text { Mikuláš }\end{array}$ \\
\hline$h_{\min }$ & 0.477 & 0.555 & 0.508 & 0.5 & 0.531 \\
\hline$T^{\text {opt }[\mathrm{min}]}$ & 67 & 294 & 67 & 77 & 196 \\
\hline$T^{\text {comp }}[\mathrm{s}]$ & 1 & 44 & 1 & 1 & 184 \\
\hline Instance: & Leopoldov & Michalovce & Nitra & $\begin{array}{c}\text { Nové } \\
\text { Zámky }\end{array}$ & Púchov \\
\hline$h_{\min }$ & 0.578 & 0.539 & 0.586 & 0.531 & 0.5 \\
\hline$T^{\text {opt }}[\mathrm{min}]$ & 75 & 383 & 135 & 472 & 117 \\
\hline$T^{\text {comp }}[\mathrm{s}]$ & 61 & 35 & 44 & 281 & 1 \\
\hline
\end{tabular}

From these overtaken instances, we created the fuzzy instances by using times $t_{i j}$ and $s_{j}$ from the original instances on the place of the main values $t^{2}{ }_{i j}$ and $s^{2}{ }_{j}$ of the triangular fuzzy times. The left and right values were obtained accordingly to the following expressions: $t_{i j}^{1}=2 t^{2}{ }_{i j} / 3, t_{i j}^{3}=4 t_{i j}^{2} / 3, s_{j}^{l}=2 s_{j}^{2} / 3$ and $s_{j}^{3}=4 s_{j}^{2} / 3$. 
The experiments were performed on a personal computer equipped with Intel Core 2 Duo with parameters: $2 \mathrm{GHz}$ and $2 \mathrm{~GB}$ RAM.

To obtain the values $F^{l}$ and $F^{2}$ in constraints (17), the procedure DejTopt was used and the associated evacuation times $F^{l}$ and $F^{2}$ are also presented in table 1 . There are also given the numbers of fleets (No. of F) and the numbers of evacuated dwelling places (No. of EP).

Table 2 and table 3 contain the following information about solving processes and obtained results:

$h_{\text {min }}$ - the highest obtained level of satisfaction,

$T^{\text {opt }}$ - the associated time of evacuation in minutes and

$T^{\text {comp }}$ - the total computational time in seconds.

Table 3: The comprehensive results obtained by the procedure FuzzyTopt_2.

\begin{tabular}{|c|c|c|c|c|c|}
\hline Instance: & Bratislava & $\begin{array}{c}\text { Dubnica nad } \\
\text { Váhom }\end{array}$ & Hrádza & Košice & $\begin{array}{c}\text { Liptovský } \\
\text { Mikuláš }\end{array}$ \\
\hline$h_{\min }$ & 0.477 & 0.555 & 0.508 & 0.5 & 0.531 \\
\hline$T^{\text {opt }}[\mathrm{min}]$ & 68 & 295 & 67 & 77 & 196 \\
\hline$T^{\text {comp }}[\mathrm{s}]$ & 0 & 43 & 0 & 0 & 67 \\
\hline Instance: & Leopoldov & Michalovce & Nitra & $\begin{array}{c}\text { Nové } \\
\text { Zámky }\end{array}$ & Púchov \\
\hline$h_{\min }$ & 0.578 & 0.539 & 0.586 & 0.531 & 0.5 \\
\hline$T^{\text {opt }}[\mathrm{min}]$ & 75 & 384 & 135 & 472 & 116 \\
\hline$T^{\text {comp }}[\mathrm{s}]$ & 24 & 4 & 11 & 86 & 0 \\
\hline
\end{tabular}

\section{Conclusions}

Two approaches to the evacuation problem under uncertainty have been introduced and compared in this paper. The first approach called FuzzyTopt_1 makes use of the iterative concept developed for the crisp evacuation problem (Janáček [3]). The second approach called FuzzyTopt_2 is directly adapted to the searching process for the maximal level of satisfaction. The both approaches have proved that they are able to solve the presented evacuation problem, in which fuzzy values conditioned by a fuzzy conditions play role. Comparing the computational results reported in table 2 and table 3 , it can be stated that the resulting times of evacuation and maximal levels of satisfaction are almost the same. Small differences in the minimal evacuations times lie within the given precision interval. As regards the computational times of the associated algorithms, the results show that the set of instances can be divided into two parts. The first part consists of so called "easy solvable" instances, where the computational times are small in general and differ only slightly for the individual algorithms. The second part of the instances is noted for longer computational times and in this case, the approach FuzzyTopt_2 completes its search approximately three times quicker. 


\section{Acknowledgement}

This work was supported by the project 'Centre of Excellence for Systems and Services of Intelligent Transport' ITMS 26220120028.

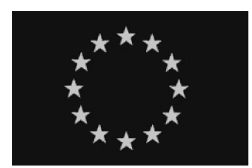

\section{Project Part-Financed by the European Union \\ European Regional \\ Development Fund}

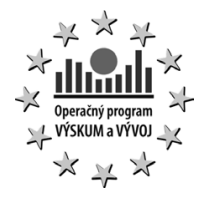

\section{References}

[1] Teichmann, D., 2008. Contribution to the Problems of Inhabitant Evacuation and Usage of Mathematical Programming for the Evacuation Planning (in Czech), Proc. Krízový management, Vol. 7, 2/2008.

[2] Janáček, J., Sibila, M., 2009. Optimal Evacuation Plan Design with IPSolver. Communications -Scientific Letters of the University of Žilina, Vol. 11, No 3, pp 29-35

[3] Janáček, J. 2009. Handling of a Non-linear Model of the Evacuation Plan Design by IP-Solver. In Proceedings of the $10^{\text {th }}$ International Symposium on Operational Research in Slovenia Nova Gorica, September 23-25, 2009, pp 279-287

[4] Teodorovič, D., Vukadinovič, K., 1998. Traffic Control and Transport Planning: A Fuzzy Sets and Neural Networks Approach. Boston: Kluwer Academic Publishers, $387 \mathrm{p}$

[5] XPRESS-MP Manual "Getting Started", 2005. Dash Associates, Blisworth, UK, p. 105.

[6] XPRESS-Mosel "User guide”, 2005. Dash Associates, Blisworth, UK, p. 99.

[7] Ramík, J., Vlach, M. (2002): Generalized Concavity in Fuzzy Optimization and Decision analysis. Kluwer Academic Publishers, Boston, $296 \mathrm{p}$ 